This is the final peer-reviewed accepted manuscript of:

J. Meng, D. Stroe, M. Ricco, G. Luo and R. Teodorescu, "A Simplified Model-Based State-of-Charge Estimation Approach for Lithium-Ion Battery With Dynamic Linear Model" in IEEE Transactions on Industrial Electronics, vol. 66, no. 10, pp. 7717-7727, Oct. 2019

The final published version is available online at:

https://doi.org/10.1109/TIE.2018.2880668

Rights / License:

The terms and conditions for the reuse of this version of the manuscript are specified in the publishing policy. For all terms of use and more information see the publisher's website.

This item was downloaded from IRIS Università di Bologna (https://cris.unibo.it/)

When citing, please refer to the published version. 


\title{
A Simplified Model based State-of-Charge Estimation Approach for Lithium-ion Battery with Dynamic Linear Model
}

\author{
Jinhao Meng, Student Member, IEEE, Daniel-Ioan Stroe, Member, IEEE, Mattia Ricco, \\ Member, IEEE, Guangzhao Luo, Member, IEEE, and Remus Teodorescu, Fellow, IEEE
}

\begin{abstract}
The performance of model based State-of-Charge (SOC) estimation method relies on an accurate battery model. Nonlinear models are thus proposed to accurately describe the external characteristics of the Lithium-ion (Li-ion) battery. The nonlinear estimation algorithms and online parameter identification methods are needed to guarantee the accuracy of the model based SOC estimation with nonlinear battery models. A new approach forming a dynamic linear battery model is proposed in this paper, which enables the application of the linear Kalman filter for SOC estimation and also avoids the usage of online parameter identification methods. With a moving window technology, Partial Least Squares (PLS) regression is able to establish a series of piecewise linear battery models automatically. One element state space equation is then obtained to estimate the SOC from the linear Kalman filter. The experiments on a $\mathrm{LiFePO}_{4}$ battery prove the effectiveness of the proposed method compared with the Extended Kalman Filter (EKF) with two Resistance and Capacitance (RC) Equivalent Circuit Model (ECM) and the Adaptive Unscented Kalman Filter (AUKF) with Least Squares Support Vector Machines (LSSVM).
\end{abstract}

Index Terms-State-of-charge estimation, partial least squares regression, Kalman filter, Lithium-ion battery.

\section{INTRODUCTION}

$\mathrm{W}$ ith the significant progress of the battery technology, Lithium-ion (Li-ion) batteries have become a promising choice for Electrical Vehicle (EV) [1] and Battery Energy Storage System (BESS) [2], [3]. The extensive usage of the Li-ion batteries is mainly because of their superior properties including long lifespan, high energy density, low self-discharge

Manuscript received June 23, 2018; revised September 10, 2018 accepted October 28,2018 . This work was supported in part by the Key Program for International S\&T Cooperation and Exchange Projects of Shaanxi Province under Grant 2017KW-ZD-05, and in part by the Fundamental Research Funds for Central Universities under Grant 3102017JC06004 and Grant 3102017OQD029. (Corresponding author: Guangzhao Luo, phone: 0086-029-88431335; fax: 0086-029-88431310; e-mail: guangzhao.luo@nwpu.edu.cn)

J. Meng and G. Luo are with the School of Automation, Northwestern Polytechnical University, Xi'an 710072, China (e-mail: scmih2008@163.com; guangzhao.luo@nwpu.edu.cn). D.-I. Stroe, M. Ricco, and R. Teodorescu are with the Department of Energy Technology, Aalborg University, Aalborg 9220, Denmark (e-mail: dis@et.aau.dk; mri@et.aau.dk; ret@et.aau.dk). rate, etc [4]. State-of-Charge (SOC) reflects the amount of energy available in a battery. In order to guarantee the effectiveness of the battery pack in real-life application, each cell needs to be balanced according to their SOCs. In addition, SOC is also an indicator in the Battery Management System (BMS) to help avoiding the overcharge and the over discharge of the cells. Nevertheless, SOC is not an inherent parameter of the battery. SOC has to be estimated because it is impossible to be directly measured by sensors.

Many SOC estimation methods have been proposed recently by researchers [5]. A straightforward way to estimate the SOC is the integration of the current flowing through the battery, which is known as the Coulomb counting method [6]. However, Coulomb counting method needs an accurate knowledge of the initial SOC, and the measurement errors from the current sensor inevitably accumulate during the calculation process of the Coulomb counting method. Open Circuit Voltage (OCV) also has the potential to reflect the SOC, which exhibits a monotonic relationship with SOC [7]. However, Li-ion battery needs a long relaxation time to gradually reach its inner equilibrium, which means accurate OCV measurement can only be obtained after hours of relaxation time as illustrated in [8]. The difficulty of the OCV measurement in real applications deteriorates its extensive usage in the SOC estimation area. Consequently, more advanced methods have been proposed to avoid the drawbacks of the previous two methods.

With the fast development of the machine learning methods, artificial intelligence-based techniques are used to estimate the SOC. Recurrent Neural Network (RNN) [9], Support Vector Machine (SVM) [10], and Multivariate Adaptive Regression Splines (MARS) [11], [12] have been used to establish a SOC estimator without the requirement of any previous knowledge of the battery electrochemistry. After collecting enough training samples in advance, those data driven based estimation methods can establish the connection between the measured signals (i.e., voltage, current, temperature) and the SOC. However, it is impractical to have the datasets covered all the working conditions of a real system since the actual conditions are unpredictable. Hence, data driven methods have difficulty in estimating an accurate SOC under the profiles that are completely different from the training dataset.

Utilizing the feedback loop structure from the control field, the model based estimation (Fig. 1) has been proposed to 
guarantee the robustness and accuracy of SOC estimation in various conditions. In Fig .1, online parameter identification aims at guaranteeing the accuracy of the battery model. The estimation algorithm is able to calculate the gain $L$ for SOC estimation according to the voltage difference $\Delta U$.

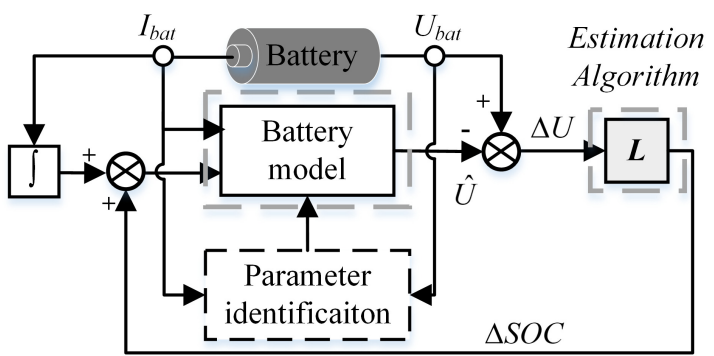

Fig. 1. Framework of the model based estimation

Compared with traditional methods, the model-based estimation methods do not require accurate knowledge of an initial SOC. As shown in Fig.1, the model-based estimation mainly contains the battery model and the estimation algorithm. PI observer [13], sliding mode observer [14], H-infinity filter [15], and Kalman filters [16]-[18], have been used as the estimation algorithms. Among them, different kinds of Kalman filters are the most frequently adopted methods in literature [5]. According to the principle of the model-based estimation, the estimation algorithm cannot handle the errors from the battery model [19]. One way to improve the performance of the battery model is integrating more affecting factors into the model equation, such as, the hysteresis behavior of OCV in $\mathrm{LiFePO}_{4}$ battery [20]. Meanwhile, the complexity of the model is also increased. It is also the main reason that the electrochemical model [21] is not as popular as Equivalent Circuit Model (ECM) in SOC estimation area. If an nonlinear model is utilized, nonlinear Kalman filter has to be used to predict the SOC [22]. Extended Kalman Filter (EKF), Unscented Kalman Filter (UKF), Particular Filter (PF) have already been applied to deal with the nonlinear battery models [5]. EKF linearizes the battery model by calculating the Jacobian matrix, but the linearization process inevitably causes an increase of the errors. Compared with EKF, more computing power is needed from the hardware when UKF and PF are used. The complex calculation of the nonlinear filters and the high order battery model reduces their values for most real-time applications. The other way to enhance the battery model is using additional online parameter identification method as shown in Fig. 1, because the parameters in the battery model change with different working conditions [23]. It should be noted that the parameters in the nonlinear battery model cannot always be identified online [24], [25]. The nonlinearities and the time scale separation in the battery model may cause some parameters only identifiable at a specific frequency [24]. The uncertainties of the results in the model-based estimation are inevitably increased because of the aforementioned issues. A suitable modeling approach, with good accuracy and less complexity, is expected in the model-based estimation. The features of the model-based estimation inspire us to simplify the battery modeling process and the complexity of the entire estimation structure while simultaneously ensuring the estimation accuracy.

Ref. [18] attempts to simplify the battery modeling process in the traditional model based estimation by using a Least Squares Support Vector Machines (LSSVM) battery model and an Adaptive Unscented Kalman Filter (AUKF). Although this method reduces the complexity of the battery model to some extent, large computational burden still remains because of the calculation of AUKF and the LSSVM modeling process. Linear Kalman filter combined with a generic Resistance and Capacitance (RC) model has already been used to estimate the SOC of a lead-acid battery in [26]. The method in [26] estimates the voltage of a bulk capacitor to form the final SOC, which essentially uses the nearly linear OCV-SOC curve of the lead-acid battery. However, in Li-ion battery, the OCV-SOC curve is nonlinear. Especially, the $\mathrm{LiFePO}_{4}$ battery has a flat OCV-SOC compared with other chemistries, which is a challenge for an accurate SOC estimation. $\mathrm{LiFePO}_{4}$ battery has a higher power density and lower cost, which is a popular choice for EVs and BESSs [27], [28]. Thus, $\mathrm{LiFePO}_{4}$ battery is also selected to validate the methods in this paper.

Partial Least Squares (PLS) regression [29], [30] is connected with a linear Kalman filter to form a simple model based SOC estimation approach in this paper. Different from previous works, this paper establishes a linear piecewise battery model online using the PLS and the moving window method. As a result, the parameters in the PLS battery model are automatically updated, and the order of the matrices in the state space function is reduced to one. SOC is then estimated by the linear Kalman filter with one order state space function. The proposed method is compared with the EKF with two RC ECM and the AUKF with LSSVM [18] in terms of estimation accuracy and execution time. The experimental results on a $\mathrm{LiFePO}_{4}$ battery prove the validation of the proposed method.

This paper is organized as follows. The PLS modeling method with moving window is introduced in Section II. A simplified SOC estimation approach including a piecewise linearized battery model and a linear Kalman filter is presented in Section III. The performance of the proposed method in term of estimation accuracy and execution time is validated in Section IV, while conclusions are given in Section V.

\section{PLS REGRESSION BASED BATTERY MODEL}

PLS regression is popular in the modeling of different industrial applications, because it captures the crucial features between the input and the output [31]. PLS has proved to be more robust than other multiple linear regression methods [32]. Therefore, PLS is chosen to dynamically linearize the battery model.

\section{A. PLS regression}

In PLS, the independent variable $X^{P L S}$ and the response $Y^{P L S}$ are decomposed into their projection and the orthogonal loading matrices as follows,

$$
\begin{aligned}
& X^{P L S}=T^{P L S} \cdot\left(P^{P L S}\right)^{T}+E_{n}^{P L S} \\
& Y^{P L S}=U^{P L S} \cdot\left(Q^{P L S}\right)^{T}+F_{n}^{P L S}
\end{aligned}
$$


where $T^{P L S}=\left[t_{1}, t_{2}, \ldots, t_{\mathrm{n}}\right]$ and $U^{P L S}=\left[u_{1}, u_{2}, \ldots, u_{\mathrm{n}}\right]$ are the score matrices, $P^{P L S}=\left[p_{1}, p_{2}, \ldots, p_{\mathrm{n}}\right]$ and $Q^{P L S}=\left[q_{1}, q_{2}, \ldots, q_{\mathrm{n}}\right]$ are the loading matrices, $E_{n}^{P L S}$ and $F_{n}^{P L S}$ are the residual terms.

Let's define $X^{P L S}=\left[x_{1}, x_{2}, \ldots, x_{\mathrm{m}}\right], Y^{P L S}=\left[y_{1}, y_{2}, \ldots, y_{\mathrm{p}}\right] .\left[t_{1}\right.$, $\left.t_{2}, \ldots, t_{\mathrm{n}}\right]$ are the dominant eigenvectors extracted from $X^{P L S}$, and $\left[u_{1}, u_{2}, \ldots, u_{n}\right]$ are the dominant eigenvectors of $Y^{P L S} . t_{1}$ is the first dominant eigenvector extracted from $X^{P L S}$, and $u_{1}$ is extracted from $Y^{P L S}$. In the process of PLS, the correlation between $t_{1}$ and $u_{1}$ is maximized at first. The regression function between $Y^{P L S}$ and $t_{1}$ is then established as $Y^{P L S}=t_{1} \cdot r_{1}+F_{1}^{P L S}$. Afterwards, the process continues with more dominant eigenvectors extracted from the residual of the regression function. The PLS process will not terminate until the regression function meets the desired precision. It's known from the calculation steps of PLS that it utilizes the advantages of the principal component analysis and the linear regression.

In order to calculate the linear factors between $X^{P L S}$ and $Y^{P L S}$ in PLS, different algorithms have been proposed. A simple concept is introduced in [33] with few calculation steps and lower computing burden. The steps are show in Fig. 2 [30]-[33] The dominant eigenvector $w_{\mathrm{k}}$ is directly calculated from the matrix $\left(X^{P L S}\right)^{\mathrm{T}} X^{P L S}\left(Y^{P L S}\right)^{\mathrm{T}} Y^{P L S}$. Then, the score matrix $T^{P L S}$ and the loading matrices $P^{P L S}$ and $Q^{P L S}$ are obtained. When the regression function is accurate enough to meet the predefined condition, the coefficient matrix $B^{P L S}$ is obtained.

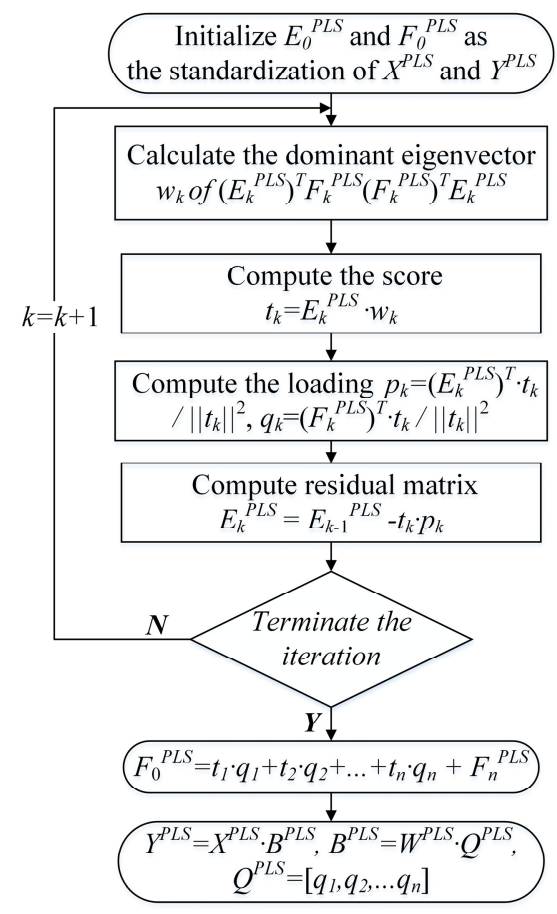

Fig. 2. The flowchart of PLS

\section{B. Battery modeling with PLS and moving window}

In order to explain the advantages of the PLS battery model with moving window, a typical battery ECM should be firstly introduced. ECM has become a popular choice in the model based estimation because of its concise structure [34]. Two RC ECM as shown in Fig. 3 has already proved to be a good tradeoff between the complexity and the accuracy. $O C V=$
$f(S O C)$ represents the nonlinear relationship between OCV and SOC. $R_{0}$ is the internal resistance, and the two RC networks represent the charge transfer $\left(R_{1}, C_{1}\right)$, the diffusion process $\left(R_{2}\right.$, $\left.C_{2}\right)$, etc.

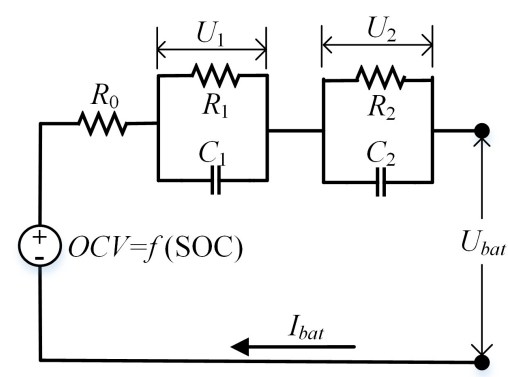

Fig. 3. Two RC ECM

The expression of the two RC ECM is as follows.

$$
\left\{\begin{array}{l}
U_{b a t}=f(S O C)-U_{1}-U_{2}-I_{b a t} \cdot R_{0} \\
I_{b a t}=\frac{U_{1}}{R_{1}}+C_{1} \frac{d U_{1}}{d t}=\frac{U_{2}}{R_{2}}+C_{2} \frac{d U_{2}}{d t}
\end{array}\right.
$$

where $U_{1}$ and $U_{2}$ are the voltages of the first and the second RC network respectively. It should be noted that the $\mathrm{RC}$ elements in the ECM are not always constant during the battery charge and discharge [23]. Hence, online parameter identification has to be used to guarantee the accuracy of the battery model. Compared with two RC ECM, the parameters in the PLS model with moving window can be automatically updated without the necessary of online parameter identification.

In this paper, the current measurement $I_{b a t}$ and the SOC are selected as the independent variable $X^{P L S}$, the terminal voltage $U_{b a t}$ is the response $Y^{P L S}$. According to the description in the previous subsection, the linear PLS battery model is expressed as the following function,

$$
U_{b a t}=B^{P L S} \cdot X^{P L S}=b_{1}+b_{2} \cdot S O C+b_{3} \cdot I_{b a t}
$$

where $B^{P L S}=\left[b_{1}, b_{2}, b_{3}\right]$ is the coefficient matrix following the steps in Fig. 2.

The relationship between the voltage and the current is nonlinear. However, it can be regarded as a linear model in a short period, which indicates the nonlinear battery model can be linearize into a series of linear PLS model. A moving window modeling method [18] is used to dynamically linearize the battery in this paper. Fig. 4 illustrates the process of establishing the PLS battery model with moving window.

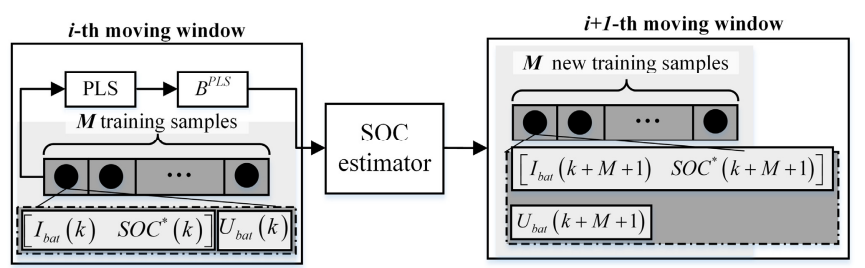

Fig. 4. The PLS based battery modeling process

The width of the moving window is defined as $M$ in Fig.4, which means that $M$ training samples are needed to calculate the $B^{P L S}$. Hence, $M$ training samples should be collected in advance to calculate the first PLS model. For the rest PLS models, the SOC in the training samples is actually the estimated SOC. The samples for the next PLS model are 
collected during the estimation process in the previous moving window. After another $M$ new training samples are collected, the PLS battery model is updated. With the application of the moving window, the PLS battery model is able to update itself with a small number of initial training samples. In addition, the PLS model is only related with the training dataset and not limited to a certain chemistry. PLS model allows the linear Kalman filter to estimate the SOC and also reduces the order of the state space function. The expression of PLS model (Eq.(4)) is simpler than the two RC ECM (Eq.(3)). However, a specific width of the moving window should be set to update the PLS battery model. The selection of a suitable width of the moving window will be discussed in Section IV.

\section{SOC ESTIMATION WITH A SIMPLIFIED STRUCTURE}

Different Kalman filters have proved to be able to estimate the battery SOC with good accuracy and robustness. Due to the nonlinearity of the ECM, nonlinear Kalman filters have been chosen to implement the SOC estimation. The computation complexity of those nonlinear Kalman filters is usually much larger than that of the linear Kalman filter. Moreover, the parameters of the battery models cannot always be effectively updated online. Instead of using nonlinear Kalman filters and online parameter identification, this section shows how to estimate SOC by linear Kalman filter and dynamic linear PLS model with a simplified structure.

\section{A. Kalman filter}

The calculation of the standard Kalman filter is introduced at first in this section as shown in Fig. 5.

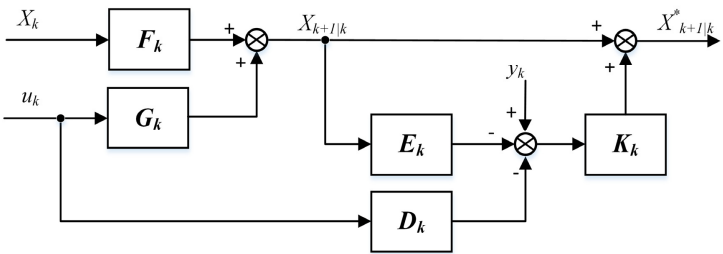

Fig. 5 Standard Kalman filter

To avoid the misleading of the symbols, the matrices in the state space equation of the Kalman filter are defined as $F_{k}, G_{k}$, $E_{k}, D_{k}$. In addition, the symbols related to EKF with two RC ECM are defined with the superscript ECM as shown in Section III.B, while the symbols in Section III.C are with the superscript Proposed Battery Model (PBM).

TABLE I

The Calculation Process of Kalman Filter

\begin{tabular}{|c|l|}
\hline \multicolumn{2}{|c|}{ Prediction } \\
\hline State Prediction & $X_{k+1 \mid k}=F_{k} \cdot X_{k}+G_{k} \cdot u_{k}$ \\
\hline Covariance Prediction & $P_{k+1 \mid k}=F_{k} \cdot X_{k} \cdot F_{k}^{T}+Q_{k}$ \\
\hline \multicolumn{2}{|c|}{ Update } \\
\hline Kalman Gain Matrix & $K_{k}=P_{k+1 \mid k} \cdot E_{k}^{T} \cdot\left(E_{k} \cdot P_{k+1 \mid k} \cdot E_{k}^{T}+R_{k}\right)^{-1}$ \\
\hline State Estimation & $X_{k+1}^{*}=X_{k+1 \mid k}+K_{k} \cdot\left(y_{k}-E_{k} \cdot X_{k+1 \mid k}-D_{k} \cdot u_{k}\right)$ \\
\hline Covariance Estimation & $P_{k+1}=\left(I-K_{k} \cdot E_{k}\right) \cdot P_{k+1 \mid k}$ \\
\hline
\end{tabular}

The gain of Kalman filter is updated to correct the estimated SOC on the foundation of the new information from the measurement and the output of the battery model. The detailed steps of the Kalman filter are shown in TABLE. I.

The calculation of the Kalman filter contains five steps. The first two steps are the state prediction and the covariance matrix prediction. The calculation of the Kalman gain matrix in the third step contains the calculation of the inverse matrix $\left(E_{k} \cdot P_{k+1 \mid k} \cdot E_{k}^{T}+R_{k}\right)^{-1}$. The order of the state space function has an effect on the calculation efficiency. In the typical two RC $\mathrm{ECM}$, the state space equation contains third-order matrices, and the calculation of the inverse matrix is time consuming.

\section{B. Extended Kalman filter with two RC ECM and Recursive Least Squares (RLS)}

A typical model based estimation with Kalman filter is detailed in this subsection to help understanding the improvement of the proposed method. EKF with two RC ECM and RLS is chosen as an example in Fig. 6.

The discretized form of the Coulomb counting equation is,

$$
\operatorname{SOC}(k+1)=\operatorname{SOC}(k)-\frac{\eta \cdot T_{\mathrm{s}}}{C_{c a p}} \cdot I_{b a t}(k)
$$

where $\eta$ is the Coulomb efficiency, $C_{\text {cap }}$ is the battery capacity, $T_{\mathrm{s}}$ is the sample time. The charge current is negative and the discharge current is positive.

According to Eq. (3) and Eq. (5), the discrete form of the state space equation of the two RC ECM is,

$$
\left\{\begin{array}{l}
X_{k+1}=F_{k}^{E C M} \cdot X_{k}+G_{k}^{E C M} \cdot u_{k}+Q_{k} \\
Y_{k+1}=E_{k}^{E C M} \cdot X_{k}+D_{k}^{E C M} \cdot u_{k}+R_{k}
\end{array}\right.
$$

where $F_{k}^{E C M}$ is a $3 \times 3$ matrix, $G_{k}^{E C M}$ is a $3 \times 1$ matrix, the $1 \times 3$ matrix $E_{k}^{E C M}$ is the Jacobian matrix. $Q_{\mathrm{k}}$ and $R_{\mathrm{k}}$ are the noise variance of the model and the measurement respectively. The details of the matrices $F_{k}^{E C M}, G_{k}^{E C M}, D_{k}^{E C M}$ and $E_{k}^{E C M}$ are shown in Fig. 6. In Fig. 6, the two RC ECM is the battery model, RLS is used as an additional parameter identification method, the gain for correcting the SOC estimation is calculated by EKF.

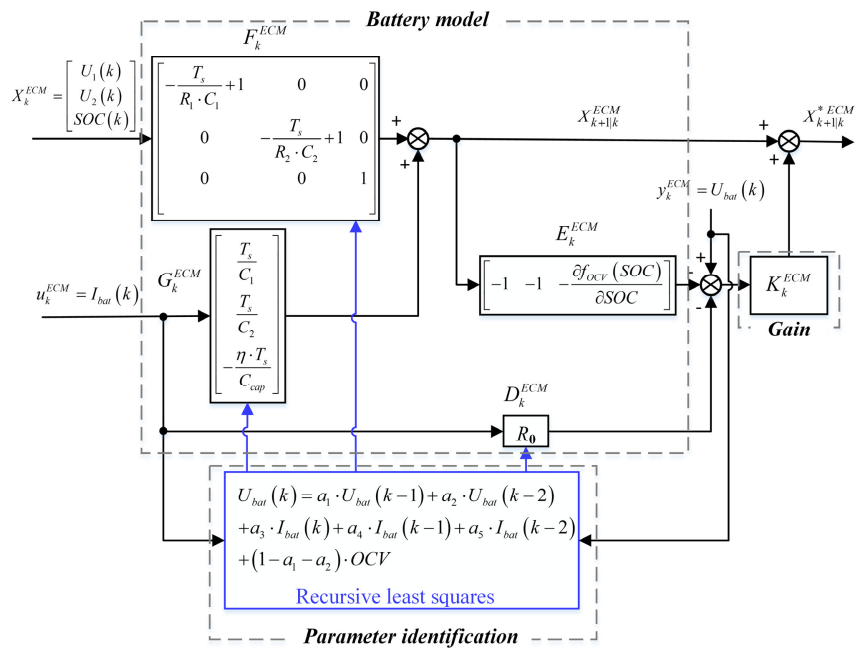

Fig. 6 Extended Kalman filter with two RC ECM and RLS 
RLS [35] is used to identify the parameters (i.e., the resistance $R_{0}, R_{1}$ and $R_{2}$, the capacitance $C_{1}$ and $C_{2}$ ) in the two RC ECM. Eq. (3) can be rewritten into the following form in RLS:

$$
\begin{aligned}
& U_{b a t}(k)=a_{1} \cdot U_{b a t}(k-1)+a_{2} \cdot U_{b a t}(k-2)+a_{3} \cdot I_{b a t}(k) \\
& +a_{4} \cdot I_{b a t}(k-1)+a_{5} \cdot I_{b a t}(k-2)+\left(1-a_{1}-a_{2}\right) \cdot O C V
\end{aligned}
$$

The calculation steps of RLS with forgetting factor are:

$$
\begin{aligned}
& \hat{y}^{R L S}(k)=\left(\varphi^{R L S}(k)\right)^{T} \theta^{R L S}(k-1) \\
& \theta^{R L S}(k)=\theta^{R L S}(k-1)+K^{R L S}(k)\left(y^{R L S}(k)-\hat{y}^{R L S}(k)\right) \\
& K^{R L S}(k)=q^{R L S}(k) \varphi^{R L S}(k) \\
& q^{R L S}(k)=\frac{P^{R L S}(k-1)}{\lambda+\left(\varphi^{R L S}(k)\right)^{T} P^{R L S}(k-1) \varphi^{R L S}(k)} \\
& P^{R L S}(k)=\frac{1}{\lambda}\left(P^{R L S}(k-1)-\right. \\
& \left.\frac{P^{R L S}(k-1) \varphi^{R L S}(k)\left(\varphi^{R L S}(k)\right)^{T} P^{R L S}(k-1)}{\lambda+\varphi^{R L S}(k) P^{R L S}(k-1) \varphi^{R L S}(k)}\right) \\
& \varphi^{\text {RLS }}(k)=\left[\begin{array}{llllll}
U_{b a t}(k-1) & U_{b a t}(k-2) & I_{b a t}(k) & I_{\text {bat }}(k-1) & I_{b a t}(k-2) & 1
\end{array}\right] \\
& \theta^{R L S}(k)=\left[\begin{array}{llllll}
a_{1} & a_{2} & a_{3} & a_{4} & a_{5} & \left(1-a_{1}-a_{2}\right) \cdot O C V
\end{array}\right]
\end{aligned}
$$

where $y^{R L S}(k)=U_{b a t}(k), \lambda$ is the forgetting factor $(0.98$ $\sim 0.995$ ). The parameters $R_{0}, R_{1}, R_{2}, C_{1}$ and $C_{2}$ in the two RC ECM are calculated from the estimated $\theta^{R L S}$ in RLS.

\section{The simplified model based estimation}

Unlike nonlinear filters, the linear Kalman filter can only work with the linear model.

According to Eq. (4) and Eq. (5), the state space function of the proposed method is established as follows,

$$
\left\{\begin{array}{c}
X_{k+1}^{P B M}=F_{k}^{P B M} \cdot X_{k}^{P B M}+G_{k}^{P B M} \cdot u_{k}^{P B M}+Q_{k}^{P B M} \\
Y_{k+1}^{P B M}=E_{k}^{P B M} \cdot X_{k}^{P B M}+D_{k}^{P B M} \cdot u_{k}^{P B M}+b 1+R_{k}^{P B M}
\end{array}\right.
$$

where $F_{k}^{P B M}=1, G_{k}^{P B M}=-\frac{\eta \cdot T_{s}}{C_{c a p}}, E_{k}^{P B M}=b_{2}, D_{k}^{P B M}=b_{3}$.

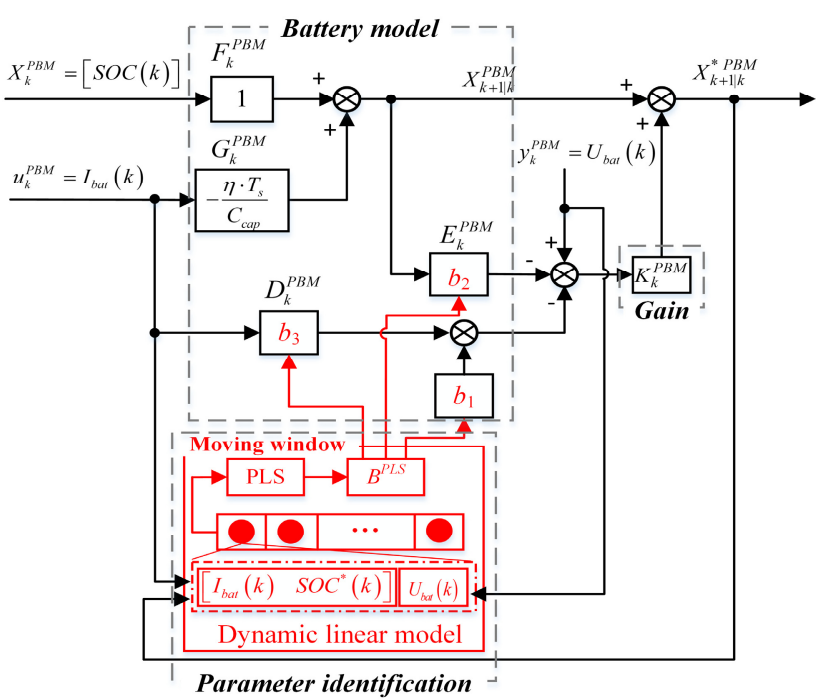

Fig. 7 The simplified model based estimation
The structure of the proposed approach is shown in Fig. 7. According to the structure in Fig. 7, the PLS is used to establish the battery model as expressed in Eq.(4), the parameters in the PLS battery model are automatically updated during the modeling process as shown in Fig. 4, the gain $K_{k}^{P B M}$ is calculated by the linear Kalman filter as described in Section III. A. Similar with the structure in Fig. 1, the proposed method in Fig. 7 achieves the function of each component in a simpler way. $E_{k}^{P B M}$ and $D_{k}^{P B M}$ are directly obtained from the coefficient matrix $B^{P L S}$ in the PLS.

The discrete form of the PLS battery model is expressed as follows,

$$
U_{b a t}(k+1)=b_{1}+b_{2} \cdot S O C(k)+b_{3} \cdot I_{b a t}(k)
$$

Accordingly, the orders of the matrices $F_{k}^{E C M}(3 \times 3), G_{k}^{E C M}$ $(3 \times 1), D_{k}^{E C M}(1 \times 3)$ and $E_{k}^{E C M}(1 \times 1)$ in the state space function of the two RC ECM are reduced to one element in the $F_{k}^{P B M}$, $G_{k}^{P B M}, D_{k}^{P B M}$ and $E_{k}^{P B M}$. There is no requirement on the calculation of the high order matrix in the Kalman filter by using the proposed method.

RLS [36], EKF [37], etc. have been used to update the parameters of ECM. However, online identification of the parameters in the ECM may lead to numerical problems and is sensitive to the measurement noise. The proposed PLS battery model is able to update the parameters with the new coming samples from the model based estimation. The identifiability of the parameters is guaranteed in various conditions.

\section{EXPERIMENTAL RESULTS}

$\mathrm{A} \mathrm{LiFePO}_{4}$ battery is used to validate the proposed method in this paper. The nominal capacity is $10 \mathrm{Ah}$ and the nominal voltage is $3.2 \mathrm{~V}$. The voltage range is from $2.0 \mathrm{~V}$ to $3.65 \mathrm{~V}$. The test bench in Fig. 8 is used to test the battery and collect the measurement. The test bench consists of a MACCOR 4000 series test station and a host computer. The test chamber provides the specific charging and discharging current profiles and also controls the ambient temperature. The measurement from MACCOR is collected by the host computer.

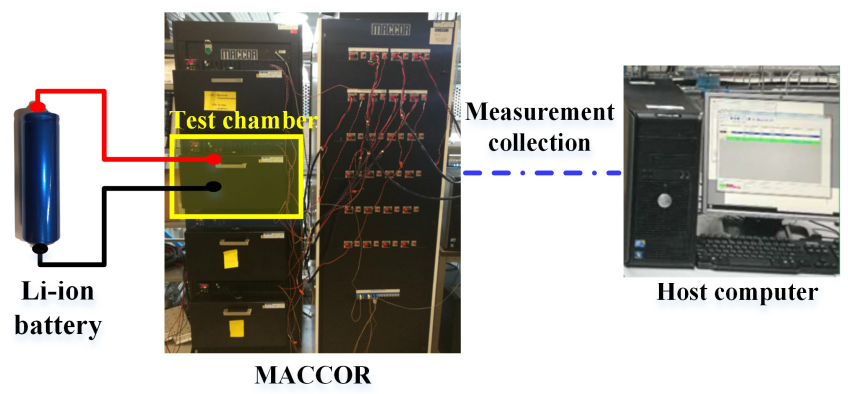

Fig. 8. The structure of the test bench

Two driving cycles, New European Driving Cycle (NEDC) [38] and Urban Dynamometer Driving Schedule (UDDS) [39], are applied to the $\mathrm{LiFePO}_{4}$ battery at first. The speed profiles of the driving cycles are transformed to the current profiles of the 
battery by using the EV model in ADVISOR [40]. The current profile is scaled down to one cell and continuously discharge the battery until its cutoff voltage is reached. The test temperature is set to $25^{\circ} \mathrm{C}$ and the sampling time is 1 second. The battery voltage and current profiles measured during NEDC and UDDS are shown in Fig. 9 with the convention that positive current means discharging.

As seen from Fig. 9, the current profile contains charge and discharge conditions and also different current amplitudes. The current in UDDS is larger in variation than that in NEDC.

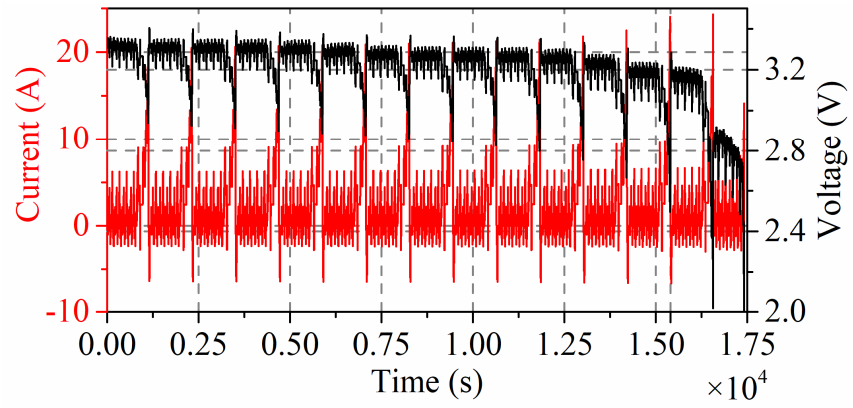

(a) NEDC

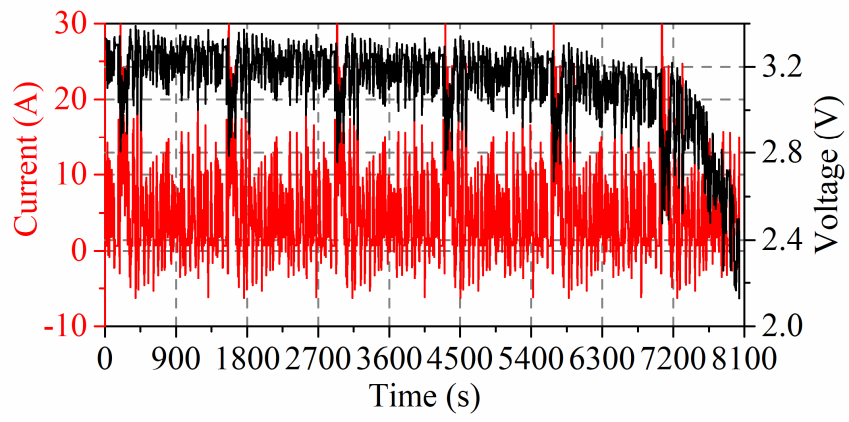

(b) UDDS

Fig. 9. Battery measurement under NEDC and UDDS

\section{A. Width selection of the moving window}

Selecting a suitable width of the moving window should be discussed before implementing the proposed approach. An example is shown in Fig. 10, the driving cycle lasts 6 seconds and the sample time is 1 second. In case 1, the width of the window is 1 second and the model is updated for six times. If the width of the moving window is set to 2 seconds as case 2 , the model updates three times. In case 3 , the model is updated two times with a 3 seconds window. Therefore, updating the PLS model with different widths of the moving window leads to the variation in the total execution time and the accuracy of the battery model in one driving cycle.

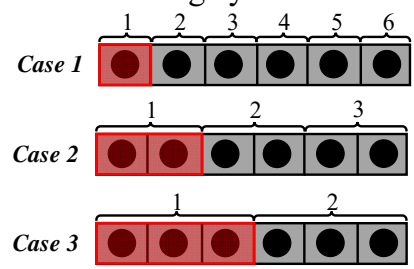

Fig. 10 Comparison of different width of the moving window
In order to select a suitable width, the relationship between the modeling accuracy and the width of the moving window is investigated. The total Mean Absolute Error (MAE) of the moving windows in one fully driving cycle is calculated to demonstrate the accuracy of the dynamic PLS model. The total MAE is calculated as,

$$
M A E=\frac{1}{N} \sum_{i=1}^{N}|\hat{x}-x|
$$

where $\hat{x}$ is the predicted voltage of the PLS model, $x$ is the voltage measurement, $N$ is the number of samples in the entire cycle.

PLS can only establish a linear model, while the battery model is essential nonlinear. A smaller moving window means the nonlinear battery model is linearized into more pieces. Therefore, the accuracy of the battery model in NEDC and UDDS increases with a smaller moving window in the test. The width of the moving window is changed from 25 seconds to 1000 seconds with a 5 seconds interval. It's clearly shown in Fig. 11 that the MAE of the battery model is increased with the width of the moving window.

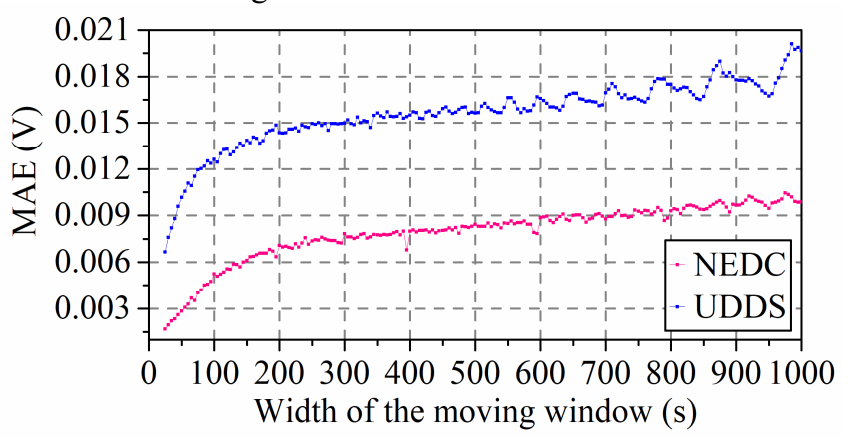

Fig. 11. The variation of MAE with different width of moving window

A larger window means the PLS model is updated in a lower frequency, and the battery model does not need to be trained so many times in one fully driving cycle. However, the numbers of the training samples in one moving window is increased with a larger window because the dimensions of the input $X^{P L S}$ and the output $Y^{P L S}$ are increased. Since the PLS battery model is only updated once in the moving window, the controller can have more computing power to implement other tasks when the model does not need to be updated. Therefore, the total execution time $t_{\text {total }}$ of the modeling method during the same driving cycle is recorded to evaluate the moving window as shown in Fig. 12. The definition of the total execution time $t_{\text {total }}$ is,

$$
t_{\text {total }}=N_{t} \cdot t_{\text {window }}
$$

where $N_{t}$ is the number of the moving windows in one fully cycle, $t_{\text {window }}$ is the execution time of each window at the updated moment.

As shown in Fig.12, $t_{\text {total }}$ decreases with a larger moving window. The decreasing rate of $t_{\text {total }}$ becomes slower with a larger window because $t_{\text {window }}$ is also increasing with the width of the window. 


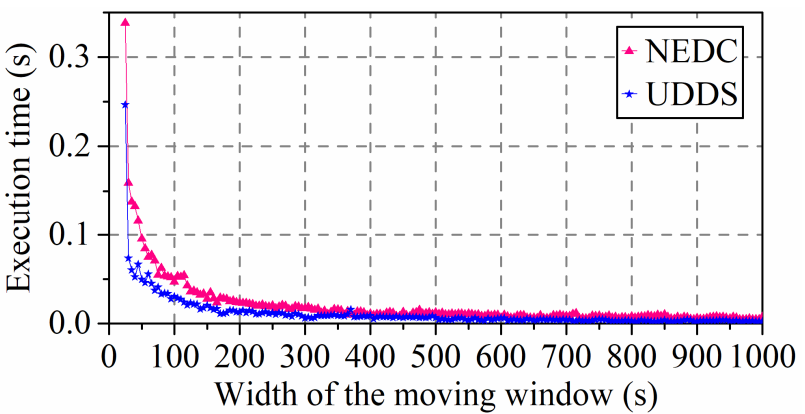

Fig. 12. The variation of $t_{\text {total }}$ with different width of moving window

Accordingly, the execution time of the proposed modeling method in the same estimation is increased with smaller moving window, while the accuracy of the battery model is also increased. Therefore, a suitable width of the moving window is a good tradeoff between the modeling accuracy and the execution time.

In Fig. 11, the increasing rate of the MAE is not so obvious when the width of the moving window is larger than 100 seconds. Moreover, the decreasing rate of the execution time also becomes slower in the same region in Fig. 12. Therefore, 100 seconds is set as the quasi-optimal width of the moving window in this paper.

\section{$B$. The performance of the PLS battery model}

When the NEDC profile is considered, the performance of the PLS battery model is shown in Fig. 13(a). The output of the PLS model is close to the voltage measurement in the entire driving cycles. The absolute errors in Fig. 13(b) are less than $0.05 \mathrm{~V}$ in most conditions and the MAE is only $0.0052 \mathrm{~V}$, which proves the accuracy of the PLS modeling with moving window.

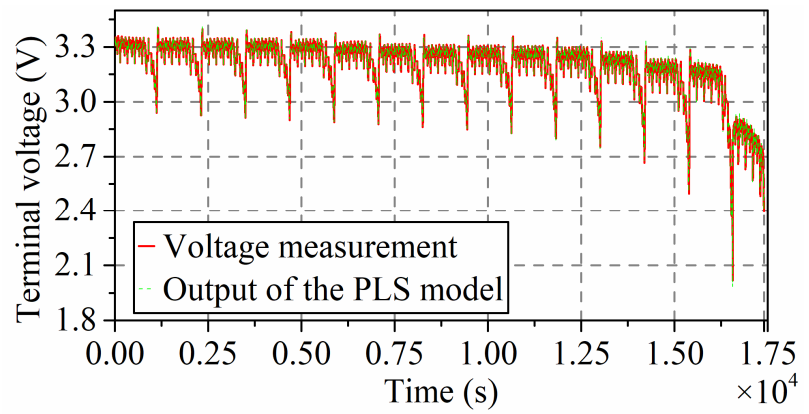

(a)

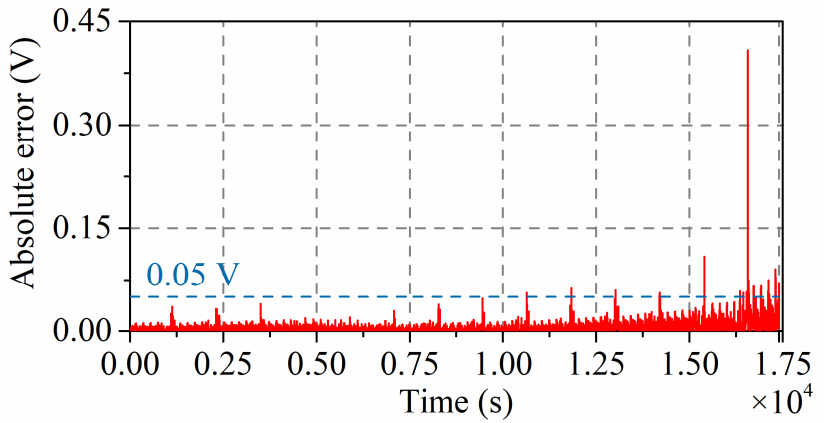

(b)

Fig. 13. The performance of the PLS battery model in NEDC
In order to validate the performance of the PLS battery model on different temperatures, NEDC is tested on $5^{\circ} \mathrm{C}, 10^{\circ} \mathrm{C}$, $25^{\circ} \mathrm{C}, 35{ }^{\circ} \mathrm{C}$ and $45{ }^{\circ} \mathrm{C}$. The modeling accuracy on five temperatures is shown in Fig. 14.

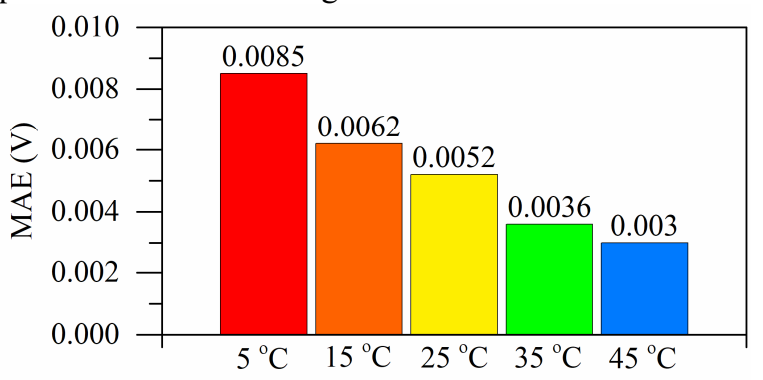

Fig. 14. The MAE of the PLS battery model

As shown in Fig. 14, the proposed PLS battery model with moving window obtains good accuracy from $5{ }^{\circ} \mathrm{C}$ to $45^{\circ} \mathrm{C}$. The modeling accuracy is better in $45^{\circ} \mathrm{C}$ than that in $5{ }^{\circ} \mathrm{C}$. Hence, the moving window could be changed to a smaller width to improve the modeling accuracy of the proposed method in lower temperature.

Despite NEDC and UDDS, three more driving cycles, Federal Test Procedure (FTP), Highway Fuel Economy Cycle (HWFET) and New York City Cycle (NYCC) are also used to verify the proposed battery model. The results in Fig. 15 prove the effectiveness of the proposed modeling method on the five driving cycles.

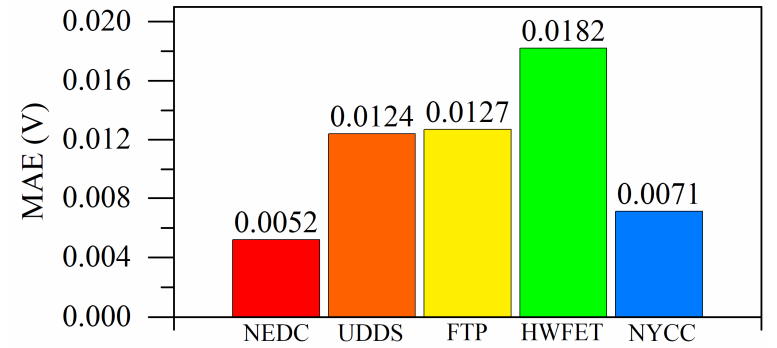

Fig. 15. MAE of the PLS battery model on different diving cycles

\section{Validation on SOC estimation}

In this section, the proposed method is compared with the AUKF with LSSVM [18] and the EKF with two RC ECM (Fig. 6). Five driving cycles, NEDC, UDDS, FTP, HWFET and NYCC are also used to verify the three SOC estimation methods in terms of the estimation accuracy and the execution time. The reference SOC comes from the MACCOR, which is based on the Coulomb counting method.

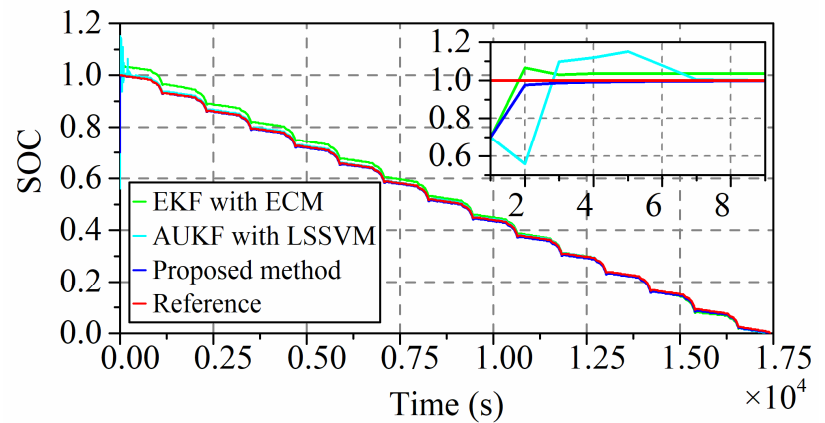

(a) SOC estimation 


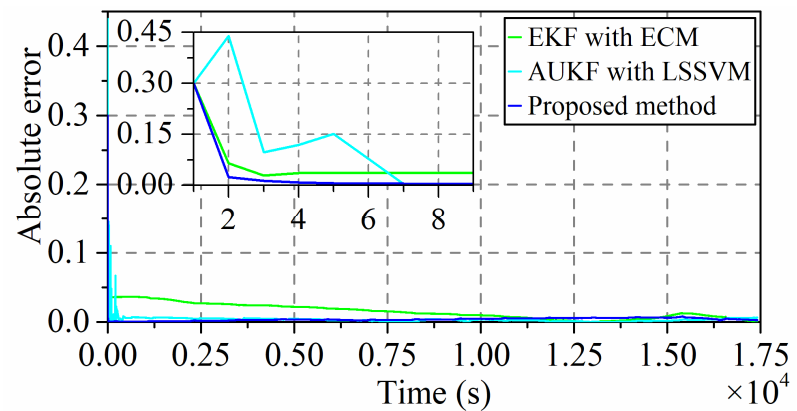

(b) Absolute error

Fig. 16. SOC estimation results in NEDC

The estimation results of the three methods in NEDC are shown in Fig. 16. The initial battery SOC is 0.7. In Fig. 16(a), all the three methods have the ability to deal with an inaccurate initial SOC. The convergence of the three algorithms is partially enlarged in Fig. 16(a). However, the estimated SOC in the proposed method and the AUKF with LSSVM converge faster and stay closer to the reference SOC. As shown in Fig. 16(b), the absolute errors of the proposed method and the AUKF with LSSVM are much smaller than that of EKF with ECM in most conditions. The MAE of EKF with ECM is 0.0151 , while the MAE is only 0.0038 for the proposed method and 0.0034 for AUKF with LSSVM. The MAE of the proposed method is around $25 \%$ of the EKF with ECM.

In UDDS, the initial SOC condition is the same as the previous experiment. The experimental results in Fig. 17(a) show that the proposed method and the AUKF with LSSVM coverage faster than the EKF with ECM. The absolute error of the proposed method is less than that of EKF in most SOC ranges. The MAE of the SOC estimation is 0.0147 for the EKF with ECM, 0.0036 for the AUKF with LSSVM and 0.0045 for the proposed method. Therefore, the proposed method also has a better accuracy than the EKF with ECM in UDDS. Since the modeling method and the estimation method are both nonlinear in AUKF with LSSVM, a better accuracy in SOC estimation is received. However, compared with the AUKF with LSSVM, the proposed method also obtains a comparable performance in estimation accuracy.

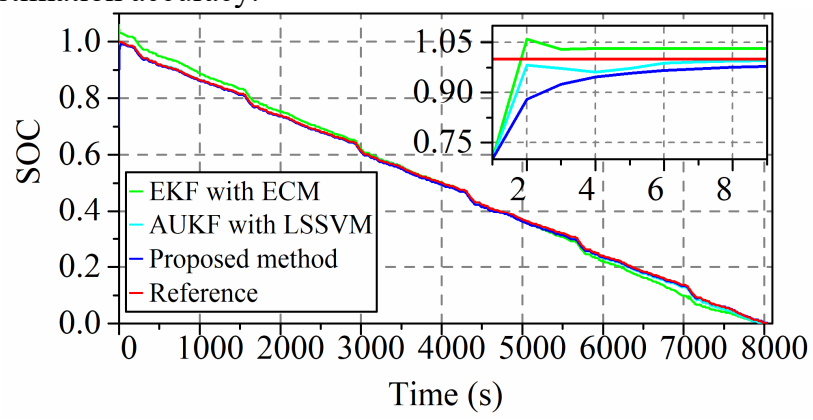

(a) SOC estimation

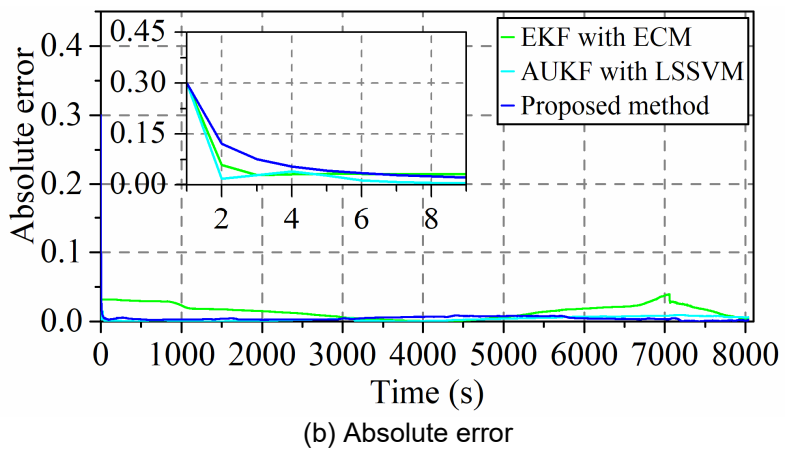

Fig. 17. SOC estimation results in UDDS

A comparison in estimation accuracy of the three methods in five driving cycles is shown in Fig. 18. AUKF with LSSVM shows the best performance in estimation accuracy, however, the proposed method obtains similar accuracy compared with the AUKF with LSSVM. The average MAE of the five driving cycles is 0.0153 for the EKF with ECM, 0.0037 for the AUKF with LSSVM, and 0.0050 for the proposed method. Therefore, the estimation accuracy proves the significant improvement of the proposed method in estimation accuracy compared with the EKF with ECM.

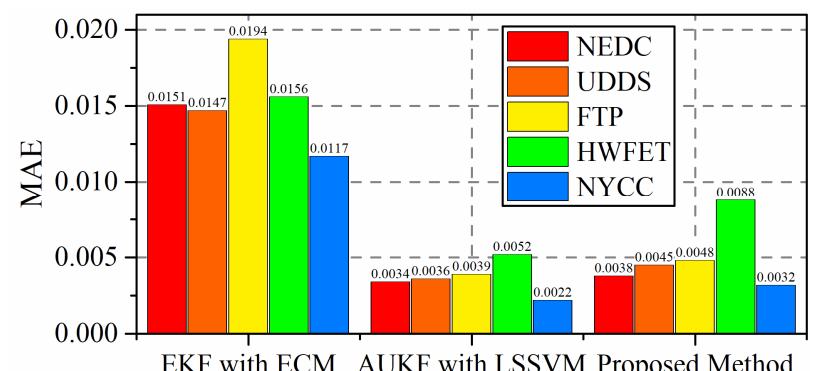

Fig. 18. Estimation accuracy of the three different methods

The computing time of the methods are verified on a PC with MATLAB $2017 \mathrm{~b}$ and $2.30 \mathrm{GHz}$ CPU. We calculate the average execution time $t_{\text {average }}$ and the maximum execution time $t_{\text {maximum }}$ of the methods in the five driving cycles as follows,

$$
\begin{aligned}
& t_{\text {average }}=\frac{1}{5}\left(t_{N E D C}+t_{U D D S}+t_{F T P}+t_{H W F E T}+t_{N Y C C}\right) \\
& t_{\text {maximum }}=\left[\begin{array}{lllll}
t_{N E D C} & t_{U D D S} & t_{F T P} & t_{H W F E T} & t_{N Y C C}
\end{array}\right]_{\max }
\end{aligned}
$$

where $t_{N E D C}, t_{U D D S}, t_{F T P}, t_{H W F E T}, t_{N Y C C}$ are the average execution time of each driving cycle.

The $t_{\text {average }}$ and $t_{\text {maximum }}$ of the three methods are shown in Fig.19. The $t_{\text {average }}$ and $t_{\text {maximum }}$ of the AUKF with LSSVM are obviously larger than the other two methods. The $t_{\text {average }}$ and $t_{\text {maximum }}$ of the proposed method are $1.46 \times 10^{-5} \mathrm{~s}$ and $2.49 \times 10^{-5} \mathrm{~s}$, which are much less than the other two methods. Accordingly, the advantage of the proposed method on the computational efficiency is proved. 


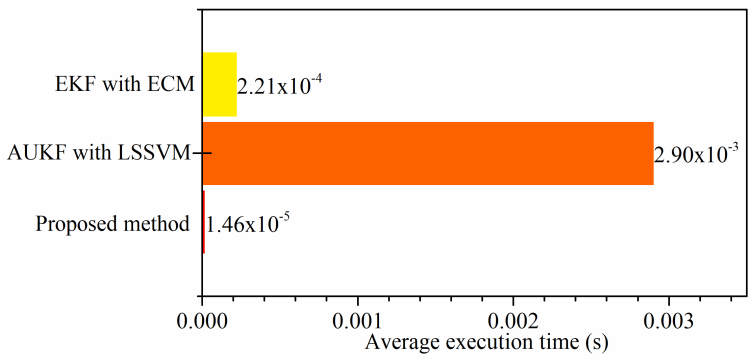

(a) Average execution time taverag

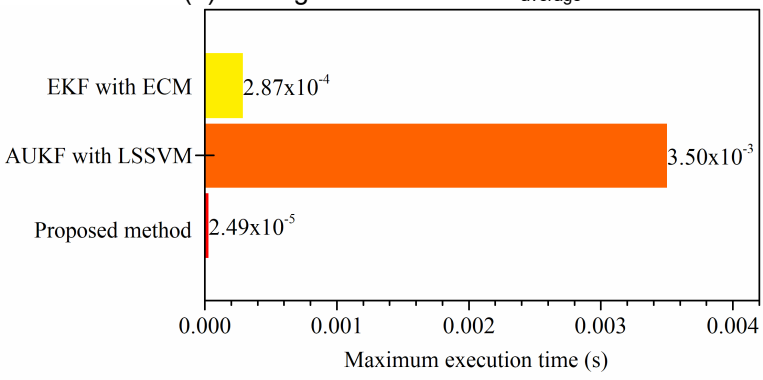

(b) Maximum execution time $t_{\text {maximum }}$

Fig. 19. The execution time of the three methods

\section{CONCLUSION}

In order to obtain an accurate battery model in the model based estimation, PLS updated with a moving window is proposed in this paper. The main advantage of the proposed method is that the state space function of the battery model is dynamically linearized into only one element, which makes it possible for the application of the linear Kalman filter. In addition, the parameters are automatically updated without the requirement of additional parameter identification methods. The order of the matrix in the state space equation is reduced from three in the EKF with ECM to one in the proposed method.

A suitable width of the moving window is discussed, and 100 seconds is proved to be a good tradeoff between the modeling accuracy and the execution time. The effectiveness of the proposed method is proved by the measurement from a $\mathrm{LiFePO}_{4}$ battery and is highlighted by comparing with the EKF with two RC ECM and the AUKF with LSSVM in five different driving cycles (NEDC, UDDS, FTP, HWFET, NYCC). The MAE for the proposed method is only $25.17 \%$ of the EKF in NEDC, and only $30.82 \%$ of the EKF in UDDS. Although AUKF with LSSVM receives the best estimation accuracy of the three, the estimation accuracy of the proposed method is also comparable to the AUKF with LSSVM. The execution time of the proposed method is much smaller than the other two methods in terms of both the average execution time $t_{\text {average }}$ and the maximum execution time $t_{\text {maximum }}$, which proves the advantages of the proposed method.

\section{REFERENCES}

[1] L. H. Saw, Y. Ye, and A. A. O. Tay, "Integration issues of lithium-ion battery into electric vehicles battery pack," J. Clean. Prod., vol. 113, pp. 1032-1045, 2016.

[2] D. I. Stroe, V. Knap, M. Swierczynski, A. I. Stroe, and R. Teodorescu, "Operation of a grid-connected lithium-ion battery energy storage system for primary frequency regulation: A battery lifetime perspective," in IEEE Transactions on Industry Applications, 2017, vol. 53, no. 1, pp. 430-438.

[3] V. Knap, R. Sinha, M. Swierczynski, D.-I. Stroe, and S. Chaudhary, "Grid inertial response with Lithium-ion battery energy storage systems," in 2014 IEEE 23rd International Symposium on Industrial Electronics (ISIE), 2014, pp. 1817-1822.

[4] B. Diouf and R. Pode, "Potential of lithium-ion batteries in renewable energy,” Renewable Energy, vol. 76. pp. 375-380, 2015.

[5] J. Meng et al., "An Overview and Comparison of Online Implementable SOC Estimation Methods for Lithium-Ion Battery," in IEEE Transactions on Industry Applications, 2018, vol. 54, no. 2, pp. $1583-1591$.

[6] L. W. Juang, P. J. Kollmeyer, R. Zhao, T. M. Jahns, and R. D. Lorenz, "Coulomb counting state-of-charge algorithm for electric vehicles with a physics-based temperature dependent battery model," in 2015 IEEE Energy Conversion Congress and Exposition, ECCE 2015, 2015, pp. 5052-5059.

[7] M. Petzl and M. A. Danzer, "Advancements in OCV measurement and analysis for lithium-ion batteries," IEEE Trans. Energy Convers., vol. 28 , no. 3, pp. 675-681, 2013.

[8] D.-I. Stroe, M. Swierczynski, A.-I. Stroe, and S. Knudsen Kær, "Generalized Characterization Methodology for Performance Modelling of Lithium-Ion Batteries," Batteries, vol. 2, no. 4, p. 37 , 2016.

[9] E. Chemali, P. J. Kollmeyer, M. Preindl, R. Ahmed, and A. Emadi, "Long Short-Term Memory Networks for Accurate State-of-Charge Estimation of Li-ion Batteries," IEEE Trans. Ind. Electron., vol. 65 , no. 8 , pp. 6730-6739, 2018

[10] J. C. Alvarez Anton, P. J. Garcia Nieto, C. Blanco Viejo, and J. A. Vilan Vilan, "Support vector machines used to estimate the battery state of charge," IEEE Trans. Power Electron., vol. 28, no. 12, pp. 5919-5926, 2013.

[11] J. C. Alvarez Anton, P. J. Garcia Nieto, E. Garcia Gonzalo, J. C. Viera Perez, M. Gonzalez Vega, and C. Blanco Viejo, “A New Predictive Model for the State-of-Charge of a High-Power Lithium-Ion Cell Based on a PSO-Optimized Multivariate Adaptive Regression Spline Approach," IEEE Trans. Veh. Technol., vol. 65, no. 6, pp. 4197-4208, Jun. 2016.

[12] J. Meng, G. Luo, E. Breaz, and F. Gao, "A robust battery state-of-charge estimation method for embedded hybrid energy system," in IECON 2015 - 41st Annual Conference of the IEEE Industrial Electronics Society, 2015, pp. 1205-1210.

[13] Jun Xu, C. C. Mi, Binggang Cao, Junjun Deng, Zheng Chen, and Siqi $\mathrm{Li}$, "The State of Charge Estimation of Lithium-Ion Batteries Based on a Proportional-Integral Observer," IEEE Trans. Veh. Technol., vol. 63, no. 4, pp. 1614-1621, May 2014.

[14] X. Chen, W. Shen, Z. Cao, and A. Kapoor, "A novel approach for state of charge estimation based on adaptive switching gain sliding mode observer in electric vehicles," J. Power Sources, vol. 246, pp. 667-678, Jan. 2014.

[15] C. Chen, R. Xiong, and W. Shen, "A Lithium-Ion Battery-in-the-Loop Approach to Test and Validate Multiscale Dual H Infinity Filters for State-of-Charge and Capacity Estimation," IEEE Trans. Power Electron., vol. 33, no. 1, pp. 332-342, Jan. 2018.

[16] G. L. Plett, "Extended Kalman filtering for battery management systems of LiPB-based HEV battery packs - Part 3. State and parameter estimation," J. Power Sources, vol. 134, no. 2, pp. 277292, Aug. 2004.

[17] Z. Chen, Y. Fu, and C. C. Mi, "State of charge estimation of lithium-ion batteries in electric drive vehicles using extended Kalman filtering," IEEE Trans. Veh. Technol., vol. 62, no. 3, pp. 1020-1030, 2013.

[18] J. Meng, G. Luo, and F. Gao, "Lithium polymer battery state-of-charge estimation based on adaptive unscented kalman filter and support vector machine," IEEE Trans. Power Electron., vol. 31, no. 3, 2016.

[19] C. Zhang, L. Y. Wang, X. Li, W. Chen, G. G. Yin, and J. Jiang, "Robust and Adaptive Estimation of State of Charge for Lithium-Ion Batteries," IEEE Trans. Ind. Electron., vol. 62, no. 8, pp. 4948-4957, 2015.

[20] X. Tang, X. Mao, J. Lin, and B. Koch, "Li-ion battery parameter estimation for state of charge," Proc. 2011 Am. Control Conf., pp. 941-946, Jun. 2011. 
[21] A. Seaman, T. S. Dao, and J. McPhee, "A survey of mathematics-based equivalent-circuit and electrochemical battery models for hybrid and electric vehicle simulation," Journal of Power Sources, vol. 256. Elsevier, pp. 410-423, 15-Jun-2014.

[22] S. Nejad, D. T. Gladwin, and D. A. Stone, "A Systematic Review of Lumped-parameter Equivalent Circuit Models for Real-time Estimation of Lithium-ion Battery States," Journal of Power Sources, vol. 316. pp. 183-196, 2016.

[23] H. He, X. Zhang, R. Xiong, Y. Xu, and H. Guo, "Online Model-based Estimation of State-of-charge and Open-circuit Voltage of Lithium-ion Batteries in Electric Vehicles," Energy, vol. 39, no. 1, pp. 310-318, 2012.

[24] A. Sharma and H. K. Fathy, "Fisher identifiability analysis for a periodically-excited equivalent-circuit lithium-ion battery model," in Proceedings of the American Control Conference, 2014, pp. 274 280.

[25] C. S. Huang and M. Y. Chow, “Accurate Thevenin's circuit-based battery model parameter identification," in IEEE International Symposium on Industrial Electronics, 2016, pp. 274-279.

[26] B. S. Bhangu, P. Bentley, D. A. Stone, and C. M. Bingham, "Nonlinear observers for predicting state-of-charge and state-of-health of lead-acid batteries for hybrid-electric vehicles," IEEE Trans. Veh. Technol., vol. 54, no. 3, pp. 783-794, 2005.

[27] M. A. Hannan, M. M. Hoque, A. Mohamed, and A. Ayob, "Review of Energy Storage Systems for Electric Vehicle Applications: Issues and Challenges," Renew. Sustain. Energy Rev., vol. 69, pp. 771-789, 2017.

[28] B. Lian, A. Sims, D. Yu, C. Wang, and R. W. Dunn, "Optimizing LiFePO4 Battery Energy Storage Systems for Frequency Response in the UK System," IEEE Trans. Sustain. Energy, vol. 8, no. 1, pp. 385 394, 2017.

[29] P. Geladi and B. R. Kowalski, "Partial least-squares regression: a tutorial," Anal. Chim. Acta, vol. 185, no. C, pp. 1-17, 1986.

[30] R. D. Tobias, "An introduction to partial least squares regression," Proc. Ann. SAS Users Gr. Int. Conf., 20th, Orlando, FL, pp. 2-5, 1995.

[31] V. Vinzi, W. Chin, J. Henseler, and H. Wang, Handbook of Partial Least Squares: Concepts, Methods and Applications. 2010.

[32] P. Geladi and B. R. Kowalski, "Partial Least Squares Regression: a Tutorial," Anal. Chim. Acta, vol. 185, no. C, pp. 1-17, 1986.

[33] S. de Jong, "SIMPLS: An Alternative Approach to Partial Least Squares Regression," Chemom. Intell. Lab. Syst., vol. 18, no. 3, pp. 251-263, 1993.

[34] R. Xiong, J. Cao, Q. Yu, H. He, and F. Sun, "Critical Review on the Battery State of Charge Estimation Methods for Electric Vehicles," IEEE Access, vol. 6, pp. 1832-1843, 2018.

[35] J. Meng et al., "Low-complexity online estimation for LiFePO4 battery state of charge in electric vehicles," J. Power Sources, vol. 395, pp. 280-288, 2018.

[36] H. Rahimi-Eichi, F. Baronti, and M. Y. Chow, "Online adaptive parameter identification and state-of-charge coestimation for lithium-polymer battery cells," IEEE Trans. Ind. Electron., vol. 61, no. 4, pp. 2053-2061, Apr. 2014.

[37] C. Hu, B. D. Youn, and J. Chung, "A multiscale framework with extended Kalman filter for lithium-ion battery SOC and capacity estimation,” Appl. Energy, vol. 92, pp. 694-704, Apr. 2012.

[38] “- Transport - UNECE.”[Online]. Available: http://www.unece.org/trans/main/wp29/wp29regs 101-120.html. [Accessed: 04-Oct-2017].

[39] "EPA Urban Dynamometer Driving Schedule (UDDS)." [Online]. Available:

https://www.epa.gov/emission-standards-reference-guide/epa-urbandynamometer-driving-schedule-udds. [Accessed: 04-Oct-2017]. “ADVISOR Advanced Vehicle Simulator." [Online]. Available: http://adv-vehicle-sim.sourceforge.net/. [Accessed: 02-Oct-2018].

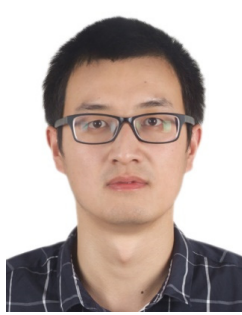

Jinhao Meng (S'14) received the M.S. degree in control theory and control engineering from Northwestern Polytechnical University (NPU), Xi'an, China, in 2013, where he is currently working toward the Ph.D. degree in electrical engineering. $\mathrm{He}$ is supported by the China Scholarship Council as a joint Ph.D. student with the Department of Energy Technology, Aalborg
University, Aalborg, Denmark, from 2017 to 2018

His research interests include battery modeling, battery states estimation, and energy management.

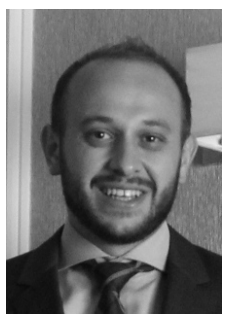

Daniel-Ioan Stroe (M'11) received the Dipl.-Ing. degree in automatics from Transilvania University of Brasov, Romania, in 2008, and M.Sc. degree in wind power systems from Aalborg University, Aalborg, Denmark, in 2010. He has been with Aalborg University since 2010, from where he obtained his Ph.D. degree in lifetime modelling of Lithium-ion batteries in 2014.

Currently, he is an Associate Professor with the Department of Energy Technology, where he leads the Battery Storage Systems research programme and the Battery Systems Testing Lab. He was a Visiting Researcher with RWTH Aachen, Germany, in 2013. He has co-authored over 90 journal and conference papers in various battery-related topics. His current research interests are in the area of energy storage systems for grid and e-mobility, Lithium-based batteries testing, modelling, diagnostics and their lifetime estimation.

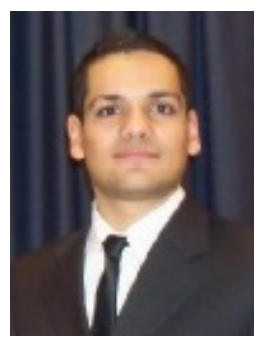

Mattia Ricco (M'16) received the master's degree (cum laude) in electronic engineering from the University of Salerno, Fisciano, Italy, in 2011, and the Ph.D. degrees in electrical and electronic engineering from the University of Cergy-Pontoise, Cergy-Pontoise, France, and in information engineering from the University of Salerno, in 2015 .

Since September 2015, he has been a Postdoctoral Research Fellow with the Department of Energy Technology at Aalborg University, Aalborg, Denmark. His main research interests include FPGA-based controllers, active battery management system, digital control of modular multilevel converters and identification algorithms for power electronics and photovoltaic systems

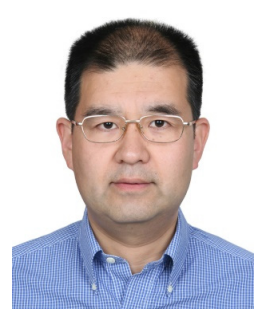

Guangzhao Luo (M'08) received the M.S. and Ph.D. degrees in electrical engineering from Northwestern Polytechnical University (NPU), Xi'an, China, in 1998 and 2003, respectively.

From 2003 to 2004, he was a Postdoctoral Research at the University of Federal Defense, Munich, Germany. He is currently a Professor with NPU. He is the Vice Director of the Rare Earth Permanent Magnet (REPM) Electric Machine and Control Engineering Center, Shaanxi Province. His research interests include advance control theory of permanent magnet electrical machine, high performance control technology of permanent magnet synchronous motor for electric traction and electric vehicle, real-time simulation technology for electrical drive system, and intelligence control of new energy conversion. Dr. Luo received the Second Prize from the China National Defense Science and Technology Progress Award in 1995 and 2011.

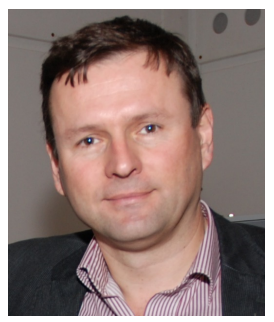

Remus Teodorescu (F'12) received the Dipl.Ing. degree in electrical engineering from the Polytechnical University of Bucharest, Bucharest, Romania, in 1989, and the Ph.D. degree in power electronics from the University of Galati, Galati, Romania, in 1994

In 1998, he joined the Power Electronics Section, Department of Energy Technology, Aalborg University, Aalborg, Denmark, where he is currently a Full Professor. Since 2013, he has been a Visiting Professor with Chalmers University. His research interests include design and control of grid-connected converters for photovoltaic and wind power systems, high voltage dc/flexible ac transmission systems based on modular multilevel converters, and storage systems based on Li-ion battery technology including modular converters and active battery management systems. 